\title{
Omalizumab for Severe Allergic Asthma: 7 Years and Open Questions
}

\author{
Markus Solèr \\ St. Claraspital, Basel, Switzerland
}

\section{Key Words}

Omalizumab · Severe allergic asthma - Treatment duration

\begin{abstract}
Anti-lgE treatment for severe allergic asthma has been available for more than seven years now. This treatment has clear clinical benefits and a good safety record. However, important questions concerning long-term dosing and treatment duration remain unanswered. This paper discusses the available information concerning the long-term use of omalizumab.

(c) 2014 S. Karger AG, Basel
\end{abstract}

Omalizumab, a humanized monoclonal antibody against human immunoglobulin E (IgE), became available in Europe and Switzerland in 2005 and 2006, respectively. It was registered for the treatment of severe allergic asthma which is insufficiently controlled by conventional treatment $[1,2]$. The concept of using a designed monoclonal antibody for the treatment of type 1 allergies provoked some doubts and hesitations during the process of registration with the European and Swiss authorities. Potent inhaled corticosteroids and long-acting $\beta_{2}$-agonists provided a highly efficient treatment for bronchial asth$\mathrm{ma}$ at the time, and the threat of severe, potentially lethal asthma attacks had lost its terror. So where should there be room for an expensive treatment in comparison with inhaled corticosteroids and long-acting $\beta$-agonists, which had to be applied subcutaneously and which would have completely new and unknown effects on the human immune system? Furthermore, what was the physiological role of IgE, the immunoglobulin class that would be neutralized by this treatment? The official European Medicines Agency registration included several restrictions, which did not correspond to the originally targeted use of the manufacturer, mainly the exclusion of moderate allergic asthma [1]. In addition to the proof of a type 1 allergy against at least one perennial antigen, the asthma patient had to have uncontrolled disease in spite of high doses of inhaled corticosteroids and long-acting $\beta_{2^{-}}$ agonists, with insufficient lung function response $\left(\mathrm{FEV}_{1}\right.$ $<80 \%)$ and symptom control (recurrent exacerbations) $[3,4]$. These limitations for the clinical use of omalizumab were only in part due to a probably rather high price of the medication, but also to nonspecified possible safety concerns related to the use of this biological agent in such a prevalent disease.

In the meantime, considerable clinical experience has accumulated with the use of this drug. Observational studies from different countries document a relevant therapeutic effect of this treatment in $70-80 \%$ of the patients in whom treatment had been attempted [5-7]. The maintenance of the effectiveness over long-term treatment has also been documented [5, 8-11]. Considering

\section{KARGER}

E-Mail karger@karger.com

www.karger.com/res
(C) 2014 S. Karger AG, Basel

0025-7931/14/0882-0158\$39.50/0
Prof. Dr. med. Markus Solèr

Chefarzt Pneumologie, St. Claraspital

Kleinriehenstrasse 30

$\mathrm{CH}-4016$ Basel (Switzerland)

E-Mail markus.soler@ claraspital.ch 
all the confounding elements in the diagnosis of severe allergic asthma $[12,13]$ and the prevalence of comorbidities in this population $[12,14]$, such a response rate has to be regarded as rather good. It seems to indicate a very good and correct workup of these patients before starting anti-IgE treatment.

The widespread clinical use of omalizumab has not confirmed fears of side effects in terms of thrombocytopenia or tumors [15]. A limited number of anaphylactic responses observed in patients during the first months of omalizumab treatment were in part timely related to the subcutaneous application. A respective black box warning has been added to the product information sheet. The frequency of such reported events is rather low compared to the frequencies reported with other monoclonal antibodies (e.g. anti-TNF), which is astonishing in a population of allergic patients with an inherent baseline incidence of anaphylactic responses. Apart from injection site irritations, the overall side effect rate seems to be rather low with this treatment.

Up to now, thousands of patients worldwide have received omalizumab treatment, some of them for more than 8 years [8]. There are several questions that come up considering the future of these patients, which can probably not be answered based on scientific data for quite some time:

- Is it correct to continue with the initially calculated dose of omalizumab even after several years of treatment?

- How long should the treatment be continued? Are there parameters that can be used to define an end of treatment?

The effectiveness of omalizumab treatment in clinical practice depends on the correct patient selection. A systematic workup of the asthma diagnosis, the allergologic diagnosis, repetitive checking and improving of the inhalation technique and checking and improving treatment compliance where necessary are central aspects [16]. In view of the costs of treatment and the considerable time and effort necessary for the application of 2- or 4-weekly injections, these factors may be even more important with omalizumab treatment than with any other asthma treatment.

For these difficult-to-treat patients in particular, there is a need for a comprehensive and systematic search for comorbidities [16, 17]. Chronic inflammatory processes in the upper airways, nose and sinuses should be diagnosed and treated optimally. Gastroesophageal reflux disease is a common comorbidity in severe asthma. Even though the treatment with proton pump inhibitors has been demonstrated to be ineffective [18], patients with this comorbidity should be systematically treated with at least an evening dose of a proton pump inhibitor in addition to applying the old-fashioned antireflux measures as far as possible, to prevent nocturnal microaspiration [19]. A high-resolution CT scan in the search for bronchiectasis and possibly a microbiological workup of sputum samples may form the basis for an antibiotic treatment, which may improve asthma control quite rapidly [17]. Candidates for omalizumab treatment should not be active smokers; this may be regarded to be a contraindication. The allergologic workup should demonstrate an IgE-type sensitization against at least one perennial antigen, as required by the drug registration authorities [3].

When omalizumab was first tested in clinical phase III trials, there was hope that blocking IgE binding to the high- and low-affinity IgE receptors might radically change the immune response to these inhaled allergens [20]. There was also hope coming up that the allergic march might be interrupted if this treatment was used early on in children. Unfortunately, the clinical studies over 1 and 2 years documented a reappearance of IgE in serum within weeks after stopping treatment [21], and usually the clinical disease reappeared at about the same intensity as before treatment [21].

An analysis of free and total IgE levels during trials in phase II and III, which were measured only in part of the study population, allowed a calculation of pharmacokinetic and pharmacodynamic (PK/PD) models [22]. These calculations indicate a slowing down of the rate of $\operatorname{IgE}$ synthesis during long-term treatment with omalizumab. The decrease in the production rate is about $50 \%$ per year [22]. We can only speculate as to the mechanism involved in this slowing of the IgE synthesis rate. It has been speculated that the prevention of IgE binding to low-affinity receptors on antigen-presenting cells might reduce their stimulatory effect on B cells, which induce this effect. For many allergic patients, e.g. those with very high IgE synthesis rates at the beginning of treatment, a $50 \%$ reduction per year means that after about 6 years of omalizumab treatment the rate of IgE synthesis has reached a level that can usually be found in healthy nonallergic individuals [22].

Astonishingly, these theoretical calculations are compatible with clinical observations in a group of 18 Swedish patients, in which omalizumab treatment was stopped after 6 years of therapy [23]. In this group of patients, asthma control was maintained with continued conventional asthma treatment after stopping the antibody injections. Even 3 years later, the clinical control was stable and the reactivity of blood basophils to the leading perennial al- 
lergens (cat dander and house dust mites) was still clearly reduced to levels below those before the start of anti-IgE treatment [24]. This is only a clinical observation in a small group of patients, but it seems to confirm the predictions from the PK/PD, model that with long-term anti-IgE treatment the rate of synthesis of new IgE antibodies seems to slow down. If these theoretical calculations and practical observations prove true, there would be two types of consequences:

1 The dosing of omalizumab in long-term treatment could be reduced according to these calculations, with relevant consequences for the cost of treatment. At the same time, the application regimen might be simplified with less injections and possibly only 4-weekly injections in patients previously treated every 2 weeks.

2 Termination of omalizumab treatment after 6 years might be considered, which would mean after about six half-lives in the reduction of the production rate of IgE. Addition to (1): One cannot expect the manufacturer of omalizumab to change the dosage regimen for this treatment based only on theoretical PK/PD calculations. On the other hand, it would be very difficult to collect new data in long-term trials to support these theoretical calculations. However, new and stringent data regarding this important question are urgently needed.

Addition to (2): Apart from the clinical observations in those 18 Swedish patients, any data regarding termination of omalizumab therapy after long-term treatment are lacking. New information regarding this aspect can be expected from the XPORT trial probably in 2014. Possibly, in a larger observational study, there would be a chance to collect more information on the cessation of long-term omalizumab treatment. For the time being, for clinical pulmonologists and allergologists treating such patients, we have to ask what would be the risk if we stop omalizumab treatment after many years.

In phase III trials and other studies over 1 and 2 years, it has repeatedly been shown that after stopping omalizumab treatment, free IgE levels in serum will increase and, in parallel, the clinical allergic symptoms will reappear [21]. The time course of this IgE reappearance corresponds to the elimination kinetics of omalizumab from serum and the rate of new IgE synthesis [21]. It has never been shown so far that there would be an overshooting IgE production or accentuation of the allergic symptoms after treatment termination. In many patients, the improved asthma control during omalizumab treatment opens a chance to maintain this state of control with conventional medications after stopping omalizumab.

If disease activity becomes very severe and a new start with omalizumab treatment is considered, the dosing of omalizumab has to continue at the same dose level as before [2]. Only if the period of interruption was longer than 12 months, a new measurement of serum total IgE will reflect the true free IgE level and may therefore be used for a new dose calculation. In any case, it would be very valuable to measure the reappearance of free $\operatorname{IgE}$ in the serum in as many patients as possible who stop omalizumab after more than 6 years of treatment. In this way, there would be a chance to collect data regarding these important questions. For the time being, it is up to the clinician and the patient to decide if they want to continue omalizumab treatment life long or if they consider a treatment stop based on this limited information.

\section{References}

1 EMEA: http://www.ema.europa.eu/docs/en_ GB/document_library/EPAR_-_Product_Information/human/000606/WC500057298. pdf.

2 Swissmedic: http://www.swissmedic.ch/zulassungen/00171/00181/00783/index.html? lang $=$ de.

3 Marcus P: Incorporating anti-IgE (omalizumab) therapy into pulmonary medicine practice. Chest 2006;129:466-474.

4 Humbert M, et al: Benefits of omalizumab as add-on therapy in patients with severe persistent asthma who are inadequately controlled despite best available therapy (GINA 2002 step 4 treatment): INNOVATE. Allergy 2005: 60:309-316.
5 Molimard M, Buhl R, Niven R, et al: Omalizumab reduces oral corticosteroid use in patients with severe allergic asthma: real-life data. Respir Med 2010;104:1381-1385.

6 Hanania N, Alpan O, Hamilos D, et al: Omalizumab in severe allergic asthma inadequately controlled with standard therapy. Ann Intern Med 2011;154:573-582.

7 Braunstahl GJ, Chen CW, Maykut R, et al: The eXpeRience registry: the 'real-world' effectiveness of omalizumab in allergic asthma. Respir Med 2013;107:1141-1151.

-8 Pace E, Ferraro M, Bruno A, et al: Clinical benefits of 7 years of treatment with omalizumab in severe uncontrolled asthmatics. J Asthma 2011;48:387-392.
9 Chen H, Eisner M, Haselkorn T, et al: Concomitant asthma medications in moderateto-severe allergic asthma treated with omalizumab. Respir Med 2013,107:60-67.

10 Menzella F, Facciolongo N, Piro R, et al: Clinical and pharmacoeconomic aspects of omalizumab: a 4-year follow-up. Ther Adv Respir Dis 2012;6:87-95.

11 Dal Negro RW, Tognella S, Pradelli L: A 36-month study on the cost/utility of add-on omalizumab in persistent difficult-to-treat atopic asthma in Italy. J Asthma 2012;49:843848.

12 Ten Brinke A, Zwindermann A, Sterk PJ, et al: Factors associated with persistent airflow limitation in severe asthma. Am J Respir Crit Care Med 2001;164:744-748. 
13 Gamble J, Stevenson M, McClean E, et al: The prevalence of nonadherence in difficult asthma. Am J Respir Crit Care Med 2009;180: 812-822.

14 Boulet LP, Boulay MẼ: Asthma-related comorbidities. Expert Rev Respir Med 2011;5: 377-393.

15 Long AA, Rahmaoui A, Rothman KJ, et al: Incidence of malignancy in omalizumab and non-omalizumab treated patients with moderate-to-severe asthma: the EXCELS Study. Am J Respir Crit Care Med 2013;187:A3869.

16 Bel EH, Sousa A, Fleming L, et al: Diagnosis and definition of severe refractory asthma: an international consensus statement from the Innovative Medicine Initiative (IMI). Thorax 2011;66:910-917.
17 Ten Brinke A, Sterk PJ, Masclee AAM, et al: Risk factors of frequent exacerbations in difficult-to-treat asthma. Eur Respir J 2005;26: 812-818.

18 American Lung Association Asthma Clinical Research Centers, Mastronarde JG, Anthonisen NR, et al: Efficacy of esomeprazole for treatment of poorly controlled asthma. N Engl J Med 2009;360:1487-1499.

19 McCallister JW, Parsons JP, Mastronarde JG: The relationship between gastroesophageal reflux and asthma: an update. Ther Adv Respir Dis 2011;5:143-150.

20 Holgate S, Casale T, Wenzel S, et al: The antiinflammatory effects of omalizumab confirm the central role of IgE in allergic inflammation. J Allergy Clin Immunol 2005;115:459465.
21 Slavin RG, Ferioli C, Tannenbaum J, et al: Asthma symptom re-emergence after omalizumab withdrawal correlates well with increasing IgE and decreasing pharmacokinetic concentrations. J Allergy Clin Immmunol 2009;123:107-113.

22 Lowe P, Renard D: Omalizumab decreases IgE production in patients with allergic (IgEmediated) asthma; PKPD analysis of a biomarker, total IgE. Br J Clin Pharmacol 2011; 72:306-320.

23 Nopp A, Johansson SGO, Ankerst J, et al: CDsens and clinical changes during withdrawal of Xolair after 6 years of treatment. Allergy 2007;62:1175-1181.

24 Nopp A, Johansson SGO, Adédoyin J, et al: After 6 years with Xolair; a 3-year withdrawal follow-up. Allergy 2007;62:1175-1181. 